
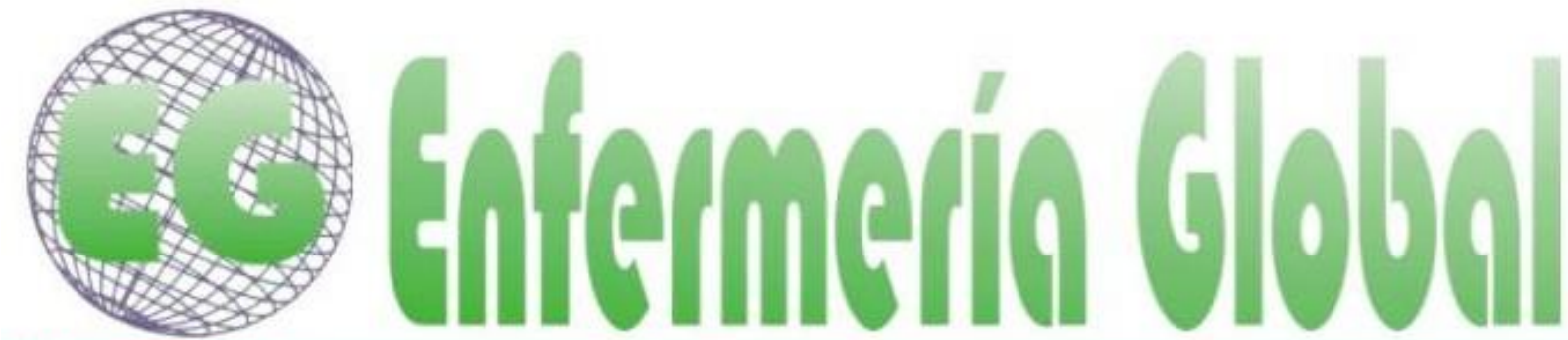

\title{
La Enfermería escolar: contenidos y percepciones sobre su pertinencia en las escuelas inclusivas
}

School nursing: contents and perceptions about its relevance in the inclusive schools

\author{
Alba-Elena Martínez-Santos ${ }^{1}$ \\ Eva Tizón-Bouza ${ }^{2}$ \\ Carmen Fernández-Morante ${ }^{3}$ \\ Lorena Casal-Otero ${ }^{4}$ \\ Beatriz Cebreiro ${ }^{5}$
}

${ }^{1}$ Doctoranda, Enfermera, Departamento de Pedagogía y Didáctica, Facultad de Ciencias de la Educación, Universidade de Santiago de Compostela. Servizo Galego de Saúde. España. alba.martinez.santos@usc.es

${ }^{2} \mathrm{PhD}$, Enfermera, Complejo Hospitalario Universitario de Ferrol, Servizo Galego de Saúde, Ferrol, España.

${ }^{3} \mathrm{PhD}$, Profesora Titular, Decana, Departamento de Pedagogía y Didáctica, Facultad de Ciencias de la Educación, Universidade de Santiago de Compostela, Santiago de Compostela, España.

${ }^{4} \mathrm{PhD}$, Profesora Ayudante Doctor, Departamento de Pedagogía y Didáctica, Facultad de Ciencias de la Educación, Universidade de Santiago de Compostela, Santiago de Compostela, España.

${ }^{5} \mathrm{PhD}$, Profesora Titular, Coordinadora de Grado, Departamento de Pedagogía y Didáctica, Facultad de Ciencias de la Educación, Universidade de Santiago de Compostela, Santiago de Compostela, España.

\section{http://dx.doi.org/10.6018/eglobal.18.4.344611}

Recibido: 4/10/2018

Aceptado: 14/01/2019

\section{RESUMEN}

Introducción: La Enfermería escolar existe en diversos países del mundo desde hace décadas. En España la presencia de Enfermería en los centros es aislada y no está regulada de manera uniforme. La promoción de la salud y Educación para la Salud juegan un papel clave en la salud actual de los escolares.

Objetivo: Determinar el grado de consenso de expertos en salud pública e infantojuvenil sobre aspectos relacionados con la Enfermería escolar para establecer los contenidos que se deben desarrollar en la función asistencial y en Educación para la Salud.

Materiales y métodos: Estudio prospectivo con enfoque cualitativo (técnica Delphi) en dos rondas con una muestra de 17 expertos. Se elaboró un cuestionario basal online pidiendo opiniones y propuestas sobre la Enfermería escolar. En la segunda ronda se incluyeron los porcentajes de respuesta y propuestas que se sometieron a priorización mediante valoración y consenso de los expertos.

Resultados: La mayoría refrendan algún tipo de presencia física de enfermeras en los centros escolares, que contribuiría a mejorar la inclusión educativa y la atención a la diversidad. Se resalta su papel en materia de promoción de la salud y prevención, identificación precoz de enfermedades y atención a la salud mental.

Conclusiones: Es preciso tener en cuenta el ámbito escolar como un nuevo y diferenciado entorno de actuación para Enfermería. El personal de Enfermería se convierte en un necesario agente de innovación en salud escolar que cuida de toda la comunidad educativa (escolares, profesorado y familia). 
Palabras clave: Servicios de Enfermería Escolar; Educación en Salud; Investigación en Educación de Enfermería; Salud Escolar; Salud Pública.

\begin{abstract}
Introduction: The School Nursing service has existed in many countries around the world for decades. In Spain, the presence of Nursing in schools is isolated and not uniformly regulated. The promotion of health and Health Education plays a key role in students' current health.

Objective: To determine experts' degree of consensus on public and child and youth health in terms of aspects related to School Nursing, with the aim of establishing the priority contents that should be developed in the care giving function and in Health Education.

Materials and methods: A two-round prospective study with a qualitative approach (the Delphi method), based on a sample of 17 experts. An online baseline questionnaire was prepared, asking for opinions and proposals on School Nursing. The second round included the response percentages and proposals subject to prioritization by means of expert assessment and consensus.

Results: Most endorse some type of physical presence of nurses in schools, which would contribute to improving educational inclusion and attention to diversity. Its role in the promotion of health and prevention, early identification of diseases, and attention to mental health is pointed out.

Conclusions: The school environment should be taken into account as a new and differentiated operating environment for Nursing. Nursing staff becomes a necessary innovation agent in school health, looking after the entire educational community (students, teachers and family).
\end{abstract}

Keywords: School Nursing; Health Education; Nursing Education Research; School health; Public Health.

\title{
INTRODUCCIÓN
}

Durante un largo e importante tiempo de la vida de nuestra infancia y juventud, durante un amplio número de horas al día, acudimos a la escuela. A lo largo de estos años, desarrollamos y acumulamos capacidades, aptitudes y actitudes fundamentales para la formación integral de nuestra personalidad. En esta etapa somos especialmente vulnerables, pues nos encontramos en un proceso de adquisición de hábitos, creencias y competencias, que nos permitirán desarrollarnos como personas ${ }^{1}$.

Hoy en día, en un mundo más complejo que nunca e inmerso en continuos cambios, el estado de salud de nuestros escolares está más expuesto que antaño. Requiere que nos planteemos una nueva estructura de atención sanitaria, que potencie nuevos enfoques de prestación de cuidados que mejoren tanto la calidad de vida de escolares sanos como de escolares con otras situaciones de salud ${ }^{(1-7)}$.

Se puede afirmar que la Educación para la Salud (EpS) es el instrumento más adecuado para prevenir malos hábitos y sus posibles consecuencias sobre la salud. Esta sirve tanto para la cura o rehabilitación, como para la prevención de enfermedades y la promoción de la salud ${ }^{(1,6,8)}$. El error a la hora de desarrollar EpS está muchas veces en la metodología utilizada para formar en estilo de vida saludable, pues para usar la más adecuada se debe conocer la EpS y saber qué implica ${ }^{9}$. Ésta es más eficaz si es de amplio alcance y establece relaciones entre el sistema de salud y el centro educativo; así como que la intervención abarque varios años escolares y se relacione con el desarrollo social y cognitivo de la población $\operatorname{diana}^{(8)}$.

La EpS en la escuela se ve sustentada por documentos de gran importancia internacional tales como la Carta de Ottawa (Organización Mundial de la Salud [OMS], 1986) ${ }^{(10)}$ que afirma que capacita a las personas para incrementar el control sobre su 
salud y, por tanto, mejorarla; o la Declaración de Yakarta (OMS, 1997) ${ }^{(11)}$ que plantea la escuela como una comunidad primordial en la EpS.

Debido a las características propias de su formación académica, las enfermeras son las profesionales más adecuadas del Sistema Nacional de Salud para llevar a cabo EpS y asistencia sanitaria continuada, y de ser necesario, inmediata ${ }^{(3-6)}$.

Enfermera escolar es aquella profesional que, dentro del ámbito del centro escolar, tiene la responsabilidad de proporcionar cuidados al alumnado de forma individualizada o coordinada dentro del equipo escolar, de modo directo, integral o individualizado. Dentro del ámbito educativo, ha de prestar un servicio que permita aumentar la capacidad del individuo y el colectivo escolar, para prevenir, detectar o tratar sus problemas de salud (Asociación Madrileña de Enfermería en Centros Educativos [AMECE], 2010) ${ }^{(12)}$.

La Enfermería escolar existe desde principios del siglo XX en diversos países de Europa, Australia o Estados Unidos. En España, sin embargo, tanto la documentación como los referentes históricos son muy escasos, así como su práctica especializada, muy reciente y aislada ${ }^{(2,3,5,13,14)}$.

Muchas de las circunstancias en relación con la salud que se producen en los centros escolares escapan al conocimiento del profesorado, ya que no se trata solo de EpS sino de atención inmediata de situaciones de salud ${ }^{(4)}$.

Las competencias profesionales de la Enfermería escolar, en España, solo han sido definidas en la Comunidad de Madrid por la Asociación Madrileña de Enfermería en Centros Educativos (AMECE, 2015) ${ }^{(15)}$, pero sería necesario establecer las prioridades en cuanto a dar mejor respuesta a las situaciones que se van produciendo en nuestra sociedad. Sus labores actuales se están viendo condicionadas por las direcciones de muchos centros al no existir una normativa que regule su actividad, creando grandes diferencias entre colegios ${ }^{(16)}$. Es por ello importante, establecer la opinión de expertos como punto de partida para discernir las principales áreas de mejora y retos a asumir por la Enfermería escolar y considerar una posible inclusión más homogénea y equitativa, que garantice el derecho a la salud por igual de nuestros escolares.

El objetivo principal de la investigación fue determinar el grado de consenso de expertos en salud pública e infantojuvenil sobre aspectos relacionados con la Enfermería escolar para establecer los contenidos prioritarios que se deben desarrollar en la función asistencial y en EpS. Los objetivos secundarios fueron conocer su opinión sobre la situación actual de la Enfermería escolar y salud escolar; establecer una propuesta en el tipo de permanencia de enfermeras en los centros escolares gallegos; e identificar las principales temáticas en EpS que debería tratar las enfermeras escolares para responder a las demandas de salud actuales.

\section{MATERIAL Y MÉTODO}

\section{Diseño}

Se llevó a cabo un estudio prospectivo con enfoque cualitativo a través de la técnica Delphi, mediante el consenso de expertos, iterativo, anónimo (evitando la influencia de los miembros dominantes del grupo) y con feedback controlado. Dicha técnica 
mezcla la evidencia científica y los valores sociales a través de la síntesis de pensamiento de un grupo especial, logrando un análisis de realidades complejas que permita impactar sobre la realidad y servir de base para una futura actuación ${ }^{(17,18)}$.

\section{Población y ámbito de estudio}

La recogida de datos se llevó a cabo en dos rondas de consulta entre marzo y mayo de 2016 a través de dos cuestionarios online, acoplados en la base del programa Google Drive ${ }^{\circledR}$, que permite el almacenamiento de archivos en línea a los que únicamente tienen acceso las investigadoras. Se hizo entrega del enlace a dichos cuestionarios a través del correo electrónico de los/as participantes, junto al cual se adjuntaba un documento informativo del estudio con el propósito de su contribución y una explicación detallada de la mecánica del proceso.

Era necesario que los/as participantes dispusieran de tiempo, interés y, sobre todo, información representativa y plural ${ }^{(17,18)}$. Para que el estudio Delphi sea el adecuado, se recomienda que el grupo de expertos personifiquen dicha pluralidad de opiniones y esté representado por personas con autoridad formal y/o un buen nivel de influencia del colectivo sanitario ${ }^{(17)}$. Para ello, se conformó el panel de expertos que se puede ver en la Tabla 1, todos ellos demostrando actividad investigadora y/o clínica vinculada a la salud pública y/o salud infanto-juvenil en España.

\begin{tabular}{|c|c|c|c|c|c|c|c|c|c|c|c|c|c|}
\hline \multirow{2}{*}{ 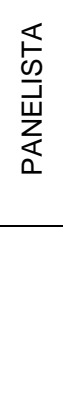 } & \multirow[t]{2}{*}{ GÉNERO } & \multirow[t]{2}{*}{$\begin{array}{l}\text { TITULACIÓN } \\
\text { SUPERIOR }\end{array}$} & \multirow[t]{2}{*}{$\begin{array}{l}\text { FORMACIÓN } \\
\text { ACADÉMICA }\end{array}$} & \multicolumn{4}{|c|}{$\begin{array}{c}\text { ÁMBITO } \\
\text { PROFESIONAL }\end{array}$} & \multicolumn{6}{|c|}{$\begin{array}{c}\text { ÁREA/S DE } \\
\text { ESPECIALIZACIÓN }\end{array}$} \\
\hline & & & & 惫 & $\sum_{\underline{m}}^{*}$ & 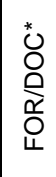 & $\begin{array}{l}\stackrel{*}{0} \\
\text { Oे } \\
\frac{0}{<}\end{array}$ & 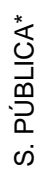 & 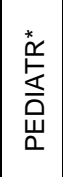 & 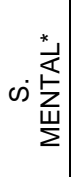 & $\begin{array}{l}* \\
\stackrel{0}{0} \\
\stackrel{2}{5}\end{array}$ & 茪 & $\begin{array}{l}\text { on } \\
\text { On } \\
\text { O } \\
\sum_{u}\end{array}$ \\
\hline 1 & FEMENINO & ENFERMERÍA & DOCTORADO & $\mathrm{X}$ & $\mathrm{X}$ & $x$ & $\mathrm{X}$ & $\mathrm{x}$ & & & & & \\
\hline 2 & FEMENINO & ENFERMERÍA & ESPECIALIDAD & $\mathrm{x}$ & $\mathrm{x}$ & $\mathrm{X}$ & $x$ & $x$ & & & & & \\
\hline 3 & FEMENINO & ENFERMERÍA & ESPECIALIDAD & $\mathrm{X}$ & $\mathrm{x}$ & & & & $x$ & & & & \\
\hline 4 & MASCULINO & MEDICINA & MÁSTER & & $\mathrm{X}$ & $x$ & & $x$ & & & & & \\
\hline 5 & FEMENINO & MEDICINA & $\begin{array}{l}\text { ESPECIALIDAD } \\
\text { /MÁSTER }\end{array}$ & $\mathrm{X}$ & $\mathrm{X}$ & & & & $\mathrm{X}$ & $\mathrm{X}$ & & & \\
\hline 6 & FEMENINO & $\begin{array}{l}\text { ENFERMERIA/ } \\
\text { PSICOLOGIA }\end{array}$ & DOCTORADO & $\mathrm{X}$ & $\mathrm{x}$ & $\mathrm{X}$ & & $\mathrm{x}$ & & $\mathrm{x}$ & & & \\
\hline 7 & FEMENINO & ENFERMERÍA & ESPECIALIDAD & $\mathrm{x}$ & & & & & $x$ & & $x$ & & \\
\hline 8 & MASCULINO & PSICOLOGÍA & MÁSTER & $x$ & $\mathrm{x}$ & $\mathrm{X}$ & $x$ & $x$ & & & & & \\
\hline 9 & FEMENINO & $\begin{array}{l}\text { ENFERMERÍA/ } \\
\text { TERAPIA } \\
\text { OCUPACIONAL }\end{array}$ & MÁSTER & $x$ & $\mathrm{x}$ & $x$ & $x$ & $x$ & $\mathrm{X}$ & & & & \\
\hline 10 & FEMENINO & ENFERMERÍA & ESPECIALIDAD & $x$ & $x$ & $x$ & & $x$ & $x$ & & & & \\
\hline 11 & FEMENINO & MEDICINA & $\begin{array}{c}\text { ESPECIALIDAD } \\
\text { /MÁSTER }\end{array}$ & $\mathrm{x}$ & $x$ & $\mathrm{x}$ & $x$ & $x$ & $\mathrm{x}$ & & & & \\
\hline 12 & FEMENINO & ENFERMERÍA & ESPECIALIDAD & $\mathrm{X}$ & & $x$ & & $x$ & & & & & \\
\hline 13 & FEMENINO & ENFERMERÍA & MÁSTER & $\mathrm{X}$ & & & $\mathrm{x}$ & $\mathrm{x}$ & & & $\mathrm{X}$ & & \\
\hline 14 & MASCULINO & $\begin{array}{l}\text { ENFERMERIA/ } \\
\text { PODOLOGÍA }\end{array}$ & ESPECIALIDAD & $\mathrm{X}$ & & & & $\mathrm{X}$ & & & $\mathrm{X}$ & & \\
\hline 15 & FEMENINO & MEDICINA & ESPECIALIDAD & $\mathrm{x}$ & $\mathrm{X}$ & & & $x$ & & & & & $x$ \\
\hline
\end{tabular}




\begin{tabular}{c|c|c|c|c|c|c|c|c|c|c|c|c|c}
\hline 16 & FEMENINO & ENFERMERÍA & ESPECIALIDAD & $\mathrm{X}$ & $\mathrm{X}$ & $\mathrm{X}$ & & $\mathrm{X}$ & & & & $\mathrm{X}$ & \\
\hline 17 & FEMENINO & ENFERMERÍA & MÁSTER & & & $\mathrm{X}$ & & $\mathrm{X}$ & & & $\mathrm{X}$ & & \\
\hline
\end{tabular}

ASISTEN: asistencial. AS/COL: Asociaciones o colegios profesionales. ENDOCR: Endocrinología y nutrición. FOR/DOC: formación/docencia. INVEST: investigación. OBS: ginecología y obstetricia. PEDIATR: pediatría. S.PÚBLICA: salud pública. S.MENTAL: salud mental. URG: urgencias y cuidados intensivos.

\section{Recogida y análisis de los datos}

En primer lugar, el equipo investigador realizó una búsqueda bibliográfica, donde se encontraron una serie de competencias y perfil de las enfermeras escolares dentro de los centros de la Comunidad de Madrid, literatura gris y diversos artículos científicos que hablan de la Enfermería escolar o salud infantojuvenil en el ámbito escolar. A partir de la información encontrada se diseñó un cuestionario basal ad hoc (C1) con 15 cuestiones sobre el fenómeno de la Enfermería escolar (Tabla 2). El C1 mantenía el equilibrio entre preguntas abiertas y cerradas. En las respuestas abiertas se dejaba espacio para texto libre y respuesta múltiple. En las respuestas cerradas, que eran de respuesta única, se etiquetaban como 1 "muy en desacuerdo" y como 5 "muy de acuerdo" (escala tipo Likert).

\begin{tabular}{|c|c|}
\hline 1 & Conocimientos en el ámbito sanitario sobre a la Enfermería escolar \\
\hline 2 & $\begin{array}{l}\text { A qué cree que es debido ese nivel de conocimiento sobre la } \\
\text { Enfermería escolar en el ámbito sanitario }\end{array}$ \\
\hline 3 & Conocimientos de la población general sobre a la Enfermería escolar \\
\hline 4 & $\begin{array}{l}\text { A qué cree que es debido ese nivel de conocimiento sobre la } \\
\text { Enfermería escolar en la población general }\end{array}$ \\
\hline 5 & Capacitación del profesorado para atender las demandas de salud \\
\hline 6 & Tratamiento transversal de la Educación para la Salud en las escuelas \\
\hline 7 & $\begin{array}{l}\text { Posible aceptación de los padres sobre la incorporación de una } \\
\text { enfermera escolar }\end{array}$ \\
\hline 8 & La inclusión de una enfermera escolar mejoraría la inclusión educativa \\
\hline 9 & $\begin{array}{l}\text { La inclusión de una enfermera escolar mejoraría la atención a la } \\
\text { diversidad }\end{array}$ \\
\hline 10 & Causas del poco impulso de la Enfermería escolar en España \\
\hline 11 & $\begin{array}{l}\text { Tipo de permanencia de las enfermeras en el centro escolar, de creerla } \\
\text { necesaria, en Galicia }\end{array}$ \\
\hline 12 & $\begin{array}{l}\text { Áreas de especialización sobre las que deben tener conocimientos las } \\
\text { enfermeras escolares }\end{array}$ \\
\hline 13 & Prioridades en la funciones de Enfermería escolar en general \\
\hline 14 & Prioridades dentro de la Educación para la Salud \\
\hline 15 & $\begin{array}{l}\text { Temáticas de mayor relevancia dentro de la promoción y } \\
\text { hábitos de vida saludable }\end{array}$ \\
\hline
\end{tabular}

Entre febrero y marzo de 2016, se pilotó el cuestionario a través de una validación del contenido por juicio de expertos. Para ello, se contactó con personas con documentada experiencia en creación de instrumentos de recogida de datos, que eran docentes universitarios. Tras realizar las modificaciones oportunas, se mostró el cuestionario a profesorado y personal sanitario, con el fin de comprobar la correcta comprensibilidad lectora y legibilidad del cuestionario. 
Toda información fue recibida y almacenada en la plataforma mencionada. Una vez concluida la primera entrega del cuestionario $\mathrm{C} 1$, se calcularon los porcentajes de respuesta y consenso de las preguntas cerradas, entendiendo que existía consenso cuando más de un $70 \%$ de los panelistas estaban de acuerdo. Se categorizaron y unificaron las respuestas a las preguntas abiertas con el fin de obtener menos grados de libertad y poder agrupar las opiniones de los participantes para un segundo envío. En el segundo cuestionario (C2) se mostraron a los expertos los resultados de las preguntas cerradas en forma de porcentaje. Se solicitó que depurasen las prioridades del conjunto inicial de propuestas categorizadas en las preguntas abiertas del $\mathrm{C} 1$. Se permitió la respuesta múltiple, de tal modo que cada panelista seleccionase aquellas cuestiones que consideraba fundamentales; excepto en las respuestas categorizadas de la pregunta 11 del cuestionario basal, donde se les pidió que se decantasen por la que creían más adecuada. Tras recibir las respuestas del $\mathrm{C} 2$, se realizó un informe con los resultados que fue enviado a los panelistas para que expresaran su aceptación o discrepancia, lo que llevaría a un tercer envío. Dicho informe fue aceptado por los expertos, dándose por cerrada la obtención de datos.

\section{Consideraciones éticas}

Se respetaron las normas éticas y legales aplicables bajo el marco ético de la Declaración de Helsinki y el Convenio de Oviedo. Se garantizó la confidencialidad y el anonimato de los participantes conforme a lo dispuesto por la Ley Orgánica 15/1999 del 13 de Diciembre sobre la Protección de Datos de Carácter Personal. Se elaboró una hoja informativa que era imprescindible haber leído y entendido antes de participar en el estudio Delphi. Se obtuvo informe favorable del comité de bioética de la Universidad de Santiago de Compostela.

\section{RESULTADOS}

Fueron invitados 17 expertos, todos aceptaron su participación en el estudio (Tabla 1). Se produjo una única pérdida en la segunda vuelta debido a la falta de tiempo del participante. El número de panelistas se situó dentro del rango establecido por esta técnica, que se encuentra entre 7 y 30 participantes, y permitiendo obtener la opinión de consenso más fiable del grupo consultado ${ }^{(17,18)}$.

El 88\% de los participantes afirmó que existe un conocimiento bajo o muy bajo sobre la Enfermería escolar en el ámbito sanitario. De la recopilación de respuestas obtenidas sobre ¿A qué cree que es debida esta situación de desconocimiento?, los participantes podían responder a varias opciones, de las que destacamos las de mayor consenso:

- La falta de regulación/promoción de una especialidad propia (81\%).

- La Ausencia de reconocimiento/regulación de los Servicios de Enfermería escolar en todas las comunidades autónomas por igual (75\%).

- La percepción de que la atención sanitaria sólo debe prestarse en instituciones sanitarias y domicilios pero no en otros ámbitos (69\%).

Todos los panelistas concordaron que el conocimiento de la Enfermería escolar en la población general es bajo o muy bajo. De la categorización surgida del C1 se les pidió que seleccionasen la principal o principales causas de dicha situación, siendo las siguientes las que mayor porcentaje alcanzaron: 
- La figura o el Servicio no está (habitualmente) presente en los colegios (94\%).

- Percepción de que la atención sanitaria solo debe prestarse en instituciones sanitarias y domicilios, pero no en otros ámbitos (81\%).

- Desconocimiento de la salud escolar por parte de la comunidad educativa (81\%).

El $94 \%$ de los expertos afirmó estar en desacuerdo o muy en desacuerdo con la capacitación del profesorado para atender las demandas de salud de su alumnado durante el horario lectivo. El $75 \%$ de los participantes estuvo en desacuerdo o muy en desacuerdo con el tratamiento transversal que se le da a la EpS en las escuelas.

Todos estuvieron de acuerdo en afirmar que la familia de los escolares valoraría positivamente la presencia de una enfermera en su centro escolar.

En cuanto a las causas del poco impulso de la Enfermería escolar, los participantes opinaron que se debe al propio desconocimiento de Servicios de Enfermería en el ámbito escolar y sus competencias (88\%), así como, por la escasa implicación de las instituciones/legisladores (69\%); siendo las dos respuestas con mayor consenso de las surgidas del $\mathrm{C} 1$.

En la primera vuelta se les pidió a los 17 panelistas que especificaran qué tipo de permanencia creían que debería tener la Enfermería escolar en los centros educativos de Galicia. De las propuestas que surgieron (Figura 1), ninguna llegó a un amplio consenso, aunque la que más obtuvo (62\%) fue la de permanecer en el centro escolar durante las horas lectivas e impartir una asignatura de EpS, teniendo un programa educativo, consulta de salud y tutorías a padres. 
Figura 1. Porcentajes de consenso a las propuestas de permanencia de Enfermería en los centros escolares.

No debe tener presencia física continua en el

colegio si no que atención primaria debe

desarrollar una relación protocolizada con la comunidad educativa

Permanencia durante las horas lectivas en cada centro, con impartición de una asignatura de

EpS y programa educativo, consulta de salud y tutorías a padres

Un mínimo de un Enfermero/a Escolar por cada 750 alumnos/as independientemente de su contexto

En función del número de alumnos/as establecido por los baremos marcados por la Red Europea de Colegios Saludables

Un enfermer@ en cada centro escolar urbano en función de los alumnos/as, donde las zonas rurales tengan un centro de referencia en la ciudad más cercana

En zonas rurales un/a Enfermero/a por centro y en zonas urbanas en centros de referencia

Al menos un Enfermer@ Escolar sin distinción entre zona rural/urbana

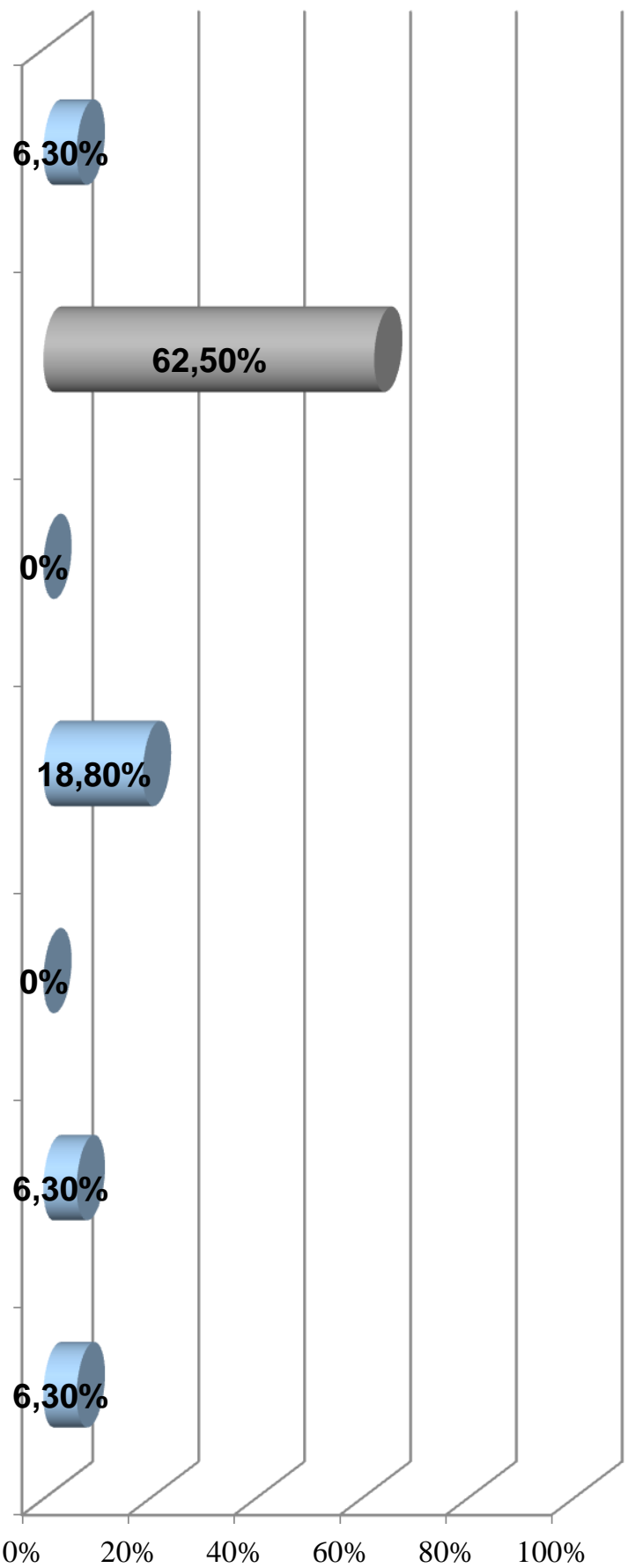

Las áreas de especialización de las que deben tener conocimientos las enfermeras escolares, según nuestro panel de expertos, alcanzaron una tasa de consenso mayor al $70 \%$, cuyos resultados específicos son los siguientes:

- Curso de Adaptación Pedagógica (conocido popularmente como el "CAP") o máster en educación equivalente (88\%). 
- Urgencias (94\%).

- Salud mental (77\%).

- Pediatría (82\%).

- Atención Primaria/Comunitaria (94\%).

De los contenidos mínimos a desarrollar dentro de las competencias de la Enfermería escolar, establecidos por consenso de las respuestas de la primera vuelta del estudio, los expertos seleccionaron los que, bajo su punto de vista profesional, son prioritarios teniendo en cuenta el contexto sociosanitario actual. Para ahondar más en el tema estudiado y obtener información más rica sobre dichas prioridades, se solicitó que las especificaran en tres grandes bloques: prioridades en la funciones de Enfermería escolar en general (Tabla 3), prioridades dentro de la EpS (Tabla 4) y temáticas de mayor relevancia dentro de la promoción y educación en hábitos de vida saludable (Tabla 5).

Tabla 3. Contenidos mínimos a desarrollar dentro de las competencias de la Enfermería escolar, por orden de prioridad.

\begin{tabular}{|c|c|c|}
\hline $\begin{array}{l}\text { № de } \\
\text { prioridad }\end{array}$ & Contenidos mínimos a desarrollar & $\begin{array}{c}\% \text { de } \\
\text { consenso }\end{array}$ \\
\hline 1 & Promoción de la salud & 87,5 \\
\hline 1 & $\begin{array}{l}\text { Prevención, identificación precoz y atención a la salud mental: } \\
\text { especial atención en casos de violencia de género, malos } \\
\text { tratos, abusos, ciberacoso, acoso escolar, diversidad sexual, } \\
\text { adicciones }\end{array}$ & 87,5 \\
\hline 2 & Participación en la programación educativa en temas de salud & 81,3 \\
\hline 3 & Generación de conocimiento científico en salud escolar & 75 \\
\hline 3 & Realización de primeros auxilios-soporte vital básico & 75 \\
\hline 4 & $\begin{array}{l}\text { Asistencia inmediata a niños/as incluidos en el programa } \\
\text { Alerta Escolar: diabetes mellitus, alergias graves con riesgo de } \\
\text { anafilaxia y epilepsia }\end{array}$ & 68,8 \\
\hline 4 & $\begin{array}{l}\text { Detección precoz de problemas sociosanitarios, del desarrollo } \\
\text { psicomotor o de anomalías }\end{array}$ & 68,8 \\
\hline 4 & $\begin{array}{l}\text { Evaluación sanitaria, nutricional y control alimenticio en } \\
\text { comedor escolar; sobre todo, en casos de alergia, celiaquía, } \\
\text { intolerancias alimentarias y diabetes }\end{array}$ & 68,8 \\
\hline 5 & Prevención de accidentes & 56,3 \\
\hline 5 & $\begin{array}{l}\text { Apoyo en los simulacros de evacuación de los centros y } \\
\text { emergencias }\end{array}$ & 56,3 \\
\hline 5 & $\begin{array}{l}\text { Trabajo activo dentro de un equipo multidisciplinar del entorno } \\
\text { educativo para establecer planes de actuación en situaciones } \\
\text { especiales como epidemias o aparición de enfermedad/es } \\
\text { rara/s }\end{array}$ & 56,3 \\
\hline 6 & $\begin{array}{l}\text { Atención en enfermedades crónicas que no están incluidas en } \\
\text { Alerta Escolar por no tener riesgo vital inminente: asma, } \\
\text { fibrosis pulmonar, enfermedades neurológicas, cardiopatías }\end{array}$ & 50 \\
\hline 6 & $\begin{array}{l}\text { Planificación individual de cuidados en alumnos con } \\
\text { patologías agudas/crónicas o necesidades de salud }\end{array}$ & 50 \\
\hline 6 & $\begin{array}{l}\text { Participación comunitaria, colaboración con entidades } \\
\text { sociales, en relación con ambientes sanos y saludables }\end{array}$ & 50 \\
\hline 6 & $\begin{array}{l}\text { Colaboración con las actividades de las Asociaciones de } \\
\text { Madres y Padres }\end{array}$ & 50 \\
\hline
\end{tabular}




\begin{tabular}{cll|c}
\hline 7 & $\begin{array}{l}\text { Primera atención en cuadros infecciosos agudos } \\
\text { (gastrointestinales, respiratorios) o fiebre, que debutan durante } \\
\text { la estancia en el colegio }\end{array}$ & 43,8 \\
\hline 8 & $\begin{array}{l}\text { Atención de múltiples urgencias y emergencias de escolares y } \\
\text { profesorado }\end{array}$ & 37,5 \\
\hline 8 & $\begin{array}{l}\text { Atención directa e información a las familias } \\
\text { Colaboración y seguimiento de programas de vacunación } \\
\text { pertinentes }\end{array}$ & 37,5 \\
\hline 8 & $\begin{array}{l}\text { Coordinación interniveles asistenciales y/o con familiares para } \\
\text { el control y seguimiento de alumnos con patologías o } \\
\text { problemas/situaciones de salud }\end{array}$ & 37,5 \\
\hline 8 & $\begin{array}{l}\text { Apoyo a los/as orientadores/as en la realización de informes } \\
\text { psicopedagógicos en lo que compete a la salud }\end{array}$ & 37,5 \\
\hline 8 & $\begin{array}{l}\text { Apoyo a las direcciones en el desarrollo de Programas de } \\
\text { Intervención }\end{array}$ & 37,5 \\
\hline 8 & $\begin{array}{l}\text { Integración en los equipos de primera intervención de los } \\
\text { Planes de Protección Escolar }\end{array}$ & 37,5 \\
\hline 8 & $\begin{array}{l}\text { Colaboración en estancias clínicas de diferentes disciplinas } \\
\text { sociosanitarias }\end{array}$ & 37,5 \\
\hline 9 & \begin{tabular}{l} 
Administración de medicación/tratamientos prescritos \\
\hline 9
\end{tabular} & $\begin{array}{l}\text { Realización de controles periódicos de salud y del desarrollo } \\
\text { Mediación en problemas en situaciones de crisis familiares } \\
\text { (situación de desamparo, familias desestructuradas...) o de } \\
\text { adaptación al medirosos } \\
\text { económicos...) }\end{array}$ & 31,3 \\
\hline 9 & $\begin{array}{l}\text { Colaboración en salidas del centro educativo de grupos de } \\
\text { escolares y profesorado }\end{array}$ & 31,3 \\
\hline 9 & $\begin{array}{l}\text { Generación de competencias de comunicación y recursos } \\
\text { sociosanitarios }\end{array}$ & 31,3 \\
\hline 10 & \begin{tabular}{l} 
Atención a niños/adolescentes dependientes \\
\hline 11
\end{tabular} & \begin{tabular}{l} 
Realización de curas, suturas y vendajes \\
\hline
\end{tabular} & 25 \\
\hline
\end{tabular}

Tabla 4. Contenidos mínimos a desarrollar dentro de las competencias de la Enfermería escolar respecto a la EpS, por orden de prioridad.

\begin{tabular}{clcc}
\hline $\begin{array}{c}\text { № de } \\
\text { prioridad }\end{array}$ & \multicolumn{1}{c}{ Contenidos mínimos a desarrollar } & $\begin{array}{c}\% \text { de } \\
\text { consenso }\end{array}$ \\
\hline 1 & $\begin{array}{l}\text { Promoción y fomento de hábitos de vida saludable desde la } \\
\text { infancia, con programas educativos adaptados a cada grupo } \\
\text { de edad }\end{array}$ & 100 \\
\hline 2 & $\begin{array}{l}\text { Formación sanitaria y Educación para la Salud a } \\
\text { profesorado, así como adiestramientos puntuales a estos en } \\
\text { patologías presentes en el centro o actuación ante } \\
\text { urgencias (reacciones anafilácticas, traumatismos, } \\
\text { hipoglucemias...) }\end{array}$ & 93,8 \\
\hline 3 & $\begin{array}{l}\text { Realización de planes de Educación para la Salud dentro de } \\
\text { un Plan de Atención Sanitaria Escolar }\end{array}$ & 87,5 \\
\hline 4 & $\begin{array}{l}\text { Educación en prevención de accidentes, o situaciones de } \\
\text { salud, en las distintas etapas de infancia o adolescencia: } \\
\text { quemaduras, caídas, ahogamientos, intoxicaciones. }\end{array}$ & 81,3 \\
\hline
\end{tabular}




\begin{tabular}{|c|c|c|}
\hline 5 & $\begin{array}{l}\text { Apoyo a los contenidos de la programación en Educación } \\
\text { para la Salud. }\end{array}$ & 68,8 \\
\hline 5 & $\begin{array}{l}\text { Formación sanitaria y Educación para la Salud de } \\
\text { familias/tutores legales (individual o grupal) }- \text { escuela de } \\
\text { familias }\end{array}$ & 68,8 \\
\hline 6 & $\begin{array}{l}\text { Educación sanitaria a niños con patologías crónicas y sus } \\
\text { familias: prevención de situaciones de riesgo según su } \\
\text { patología; identificación de los primeros síntomas de } \\
\text { reagudización de su enfermedad; uso correcto de } \\
\text { dispositivos necesarios para tratar su enfermedad: } \\
\text { inhaladores, glucómetros, bolígrafos de insulina, auto } \\
\text { inyectores de adrenalina (para los más mayores), } \\
\text { dispositivos de fisioterapia respiratoria... }\end{array}$ & 62,5 \\
\hline 7 & $\begin{array}{l}\text { Asignatura de Educación para la Salud, al menos cada } 3 \\
\text { años, sobre aspectos relevantes para cada edad }\end{array}$ & 56,3 \\
\hline 7 & $\begin{array}{l}\text { Colaboración con las actividades de las Asociaciones de } \\
\text { Padres y Madres en materia de Educación para la Salud }\end{array}$ & 56,3 \\
\hline 8 & $\begin{array}{l}\text { Formación a los alumnos respecto a posibles patologías que } \\
\text { puedan sufrir compañeros (atención a urgencias o cuidados) }\end{array}$ & 50 \\
\hline 9 & $\begin{array}{l}\text { Formación y actualización de temas de interés sobre salud y } \\
\text { autocuidados dirigidos a toda la comunidad educativa }\end{array}$ & 43,8 \\
\hline 10 & $\begin{array}{l}\text { Educación emocional y actividades psicoeducativas, con la } \\
\text { enfermeras integradas en gabinete de psicología y } \\
\text { orientación: identificar emociones, saber verbalizarlas, } \\
\text { fomentar la sensibilidad, la empatía, el respeto, la tolerancia } \\
\text { y la diversidad }\end{array}$ & 37,5 \\
\hline 11 & $\begin{array}{l}\text { Derechos y deberes de los/as pacientes dirigido a } \\
\text { profesorado y familias }\end{array}$ & 31,3 \\
\hline 11 & $\begin{array}{l}\text { Salud comunitaria: el papel del individuo como mediador en } \\
\text { materia de salud }\end{array}$ & 31,3 \\
\hline
\end{tabular}

Tabla 5. Temáticas de especial prioridad establecidas por consenso en cuanto a promoción y educación de hábitos de vida saludable.

\begin{tabular}{c|l|c}
\hline $\begin{array}{c}|c| \\
\text { № de } \\
\text { prioridad }\end{array}$ & \multicolumn{1}{|c}{ Temática } & $\begin{array}{c}\% \text { de } \\
\text { consenso }\end{array}$ \\
\hline 1 & $\begin{array}{l}\text { Hábitos tóxicos/adicciones: alcoholismo, tabaquismo, otras } \\
\text { drogas (incidiendo especialmente en la adolescencia) }\end{array}$ & 100 \\
\hline 1 & $\begin{array}{l}\text { Higiene y salud afectivo-sexual (sobre todo en las edades } \\
\text { más comprometidas como son la pre/adolescencia): } \\
\text { sexualidad responsable, infecciones de transmisión sexual, } \\
\text { embarazo, violencia/rol de género }\end{array}$ & 100 \\
\hline 2 & $\begin{array}{l}\text { Nutrición: hábitos alimentarios, alergias alimentarias, } \\
\text { obesidad, malnutrición }\end{array}$ & 93,8 \\
\hline 2 & $\begin{array}{l}\text { Ejercicio físico: práctica de deporte, sedentarismo e } \\
\text { importancia del descanso }\end{array}$ & 93,8 \\
\hline 2 & $\begin{array}{l}\text { Prevención y mediación en la salud mental: conductas de } \\
\text { riesgo, trastornos de alimentación y comportamiento }\end{array}$ & 93,8 \\
\hline 2 & $\begin{array}{l}\text { Primeros auxilios/RCP básicos en todas las etapas de la } \\
\text { vida. RCP con desfibrilador semiautomático para } \\
\text { alumnos/as de secundaria, profesorado y familia }\end{array}$ & 93,8 \\
\hline 3 & \begin{tabular}{l} 
Higiene personal, lavado de manos y salud bucodental \\
\hline
\end{tabular}
\end{tabular}




\begin{tabular}{c|l|c}
\hline 4 & Hábitos personales & 81,3 \\
\hline 5 & Riesgos y peligros: traumatismos, educación vial... & 68,8 \\
\hline 5 & Maltrato, acoso escolar & 68,8 \\
\hline 6 & Adherencia a la vacunación & 50 \\
\hline 7 & Enfermedad, secuelas y diversidad funcional/dependencia & 43,8 \\
\hline 7 & Redes sociales, internet, utilización sana de las TIC & 43,8 \\
\hline 8 & $\begin{array}{l}\text { Enfermedades crónicas (respiratorias, renales, hepáticas, } \\
\text { reumatológicas, cardiológicas, inmunodeficiencias...) }\end{array}$ & 31,3 \\
\hline 8 & Empatía y asertividad & 31,3 \\
\hline 9 & Estrés, dirigido a profesorado y familias & 25 \\
\hline
\end{tabular}

\section{DISCUSIÓN}

Hace unos años, a pesar de la consolidación de los servicios de Enfermería escolar en otros países, no se contemplaba que en nuestros centros escolares fuera necesaria la permanencia continuada o parcial de ningún profesional sanitario ${ }^{(19)}$. Sin embargo, en los últimos tiempos, han surgido diversas iniciativas en algunas comunidades autónomas de nuestro país, difundidas a través de colegios profesionales y asociaciones a través de sus revistas y páginas web. Es el caso del colegio de enfermería de Madrid y la Asociación Madrileña de Enfermería en Centros Educativos y o Asociación Diabetes Madrid. Ésta última incluso ofrece una lista de los colegios de su comunidad, tanto de titularidad pública como privada, donde se reflejan los servicios de enfermería escolar disponibles.

A pesar de estos nuevos impulsos en ciertas regiones, la literatura científica en materia de Enfermería escolar en España, así como investigaciones sobre planes de salud escolar, son escasas, generalistas y sin aplicación práctica. En Galicia, no existe ninguna bolsa pública de Enfermería escolar; su particular dispersión poblacional hace que la labor presencial de las enfermeras en los centros se dificulte.

Un informe del Ministerio de Sanidad, Servicios Sociales e Igualdad de $2013^{(20)}$ ha rescatado la situación de estancamiento, e incluso empeoramiento, en ciertos estilos de vida durante la infancia y adolescencia en España. Esto hace necesario priorizar acciones en aquellos ámbitos que respondan a las necesidades existentes a nivel local ${ }^{(21)}$, reforzando la idea de un estudio más pormenorizado sobre la situación social y sanitaria de las diferentes regiones.

En la mayoría de centros escolares es el profesorado el que, en caso de necesidad sanitaria, asiste al alumnado. Muchos de ellos lo ven arriesgado debido a su falta de formación y no se sienten capacitados para atender las demandas de salud de su alumnado ${ }^{(3,22,23)}$; opinión en la que también concuerdan nuestros panelistas.

La familia, parte fundamental de la comunidad escolar ${ }^{(24)}$, valora muy positivamente los cuidados de Enfermería, situándolos en "segundo lugar entre algunos servicios escolares que hipotéticamente se podrían prestar, y en primer lugar si los que responden son los progenitores de escolares con enfermedades crónicas" (19)

Algunos escolares con enfermedades se ven en la obligación de tener que faltar a clase 0 abandonar el colegio, ya que no hay un profesional cualificado para atender sus necesidades (25). En muchas ocasiones, cuando regresan, tienen serias 
dificultades para seguir el ritmo de las clases y perciben poco apoyo para normalizar su situación ${ }^{(26)}$. En este sentido, todos los expertos de nuestro estudio afirman que la presencia de enfermeras mejoraría la inclusión educativa y la atención a la diversidad en la infancia y adolescencia. Comparten estas afirmaciones González García y López Langa ${ }^{(2)}$, que afirman que las enfermeras en las escuelas normalizan la vida de los escolares con alguna enfermedad. Además de favorecer la continuación de sus estudios reglados, facilita la conciliación familiar, tal como manifiesta en un estudio de 2015 de la Fundación Diabetes Madrid que afirma que el 60\% de los progenitores han tenido que modificar su actividad laboral. Máxime hoy en día que la mujer ya no solo trabaja dentro del ámbito del hogar.

La presencia de enfermeras escolares es una necesidad sentida por la comunidad escolar ${ }^{(3,23)}$. Muchos autores coinciden también en que su incorporación mejoraría la calidad de vida en la infancia y adolescencia $(2,3,13,25)$; concordando con los resultados del informe final del grupo Delphi. Este último punto cobra especial importancia, pues, un estudio sobre factores relacionados con la calidad de vida en la adolescencia determina que el funcionamiento familiar, el sexo masculino, la actividad física y las enfermedades/diversidad funcional son elementos protectores. Además, ciertos hábitos se asocian a una disminución de esta calidad de vida, los cuales se podrían trabajar a través de la promoción de la salud escolar ${ }^{(27)}$. Ámbito donde las enfermeras juegan un papel clave en colaboración con la comunidad educativa (profesorado, familias y alumnado) ${ }^{(28)}$.

En vista de lo rescatado en la literatura, junto con todos los contenidos a desarrollar resultado del estudio Delphi, se podría afirmar que, en el plano asistencial, la necesidad de inclusión de una enfermera escolar está más que sustentada. Asimismo, se vería justificado el seguimiento periódico de la salud del alumnado, pudiendo recopilarse información que no éramos capaces de ver o analizar hasta el momento. Situación de gran importancia durante la adolescencia, que acude de manera muy poco frecuente a la consulta de Atención Primaria ${ }^{(4,25,28,29)}$.

Un informe Ministerial ${ }^{(21)}$ considera especialmente importante la esfera de la salud mental, ya que los ámbitos de bienestar, seguridad y salud emocional requerirían mucha mayor atención de la que reciben actualmente. En la actualidad, se sigue sin dar la importancia que realmente se merece a la distribución de los determinantes de salud, ya que no se invierte lo necesario en inculcar hábitos de vida saludables ${ }^{(9)}$. Los participantes en nuestro estudio destacan todos aquellos aspectos de promoción de la salud y prevención de la enfermedad, más allá de las actividades puramente asistenciales, que se sitúan en los últimos lugares en cuanto a la prioridad. Y, es que, tal y como dice Gallego de Pardo ${ }^{(1)}$, no es nada nuevo considerar que las intervenciones en EpS bien implantadas en el ámbito escolar repercutirán en una reducción de problemas sociosanitarios en el futuro, teniendo un impacto en la economía de las naciones. A esta sentencia, Riquelme Pérez ${ }^{(8)}$ añade que "esto es así, especialmente, si dichos programas se sustentan y apoyan la tarea básica de los centros de enseñanza y si están bien conectados con la familia y la comunidad local"; aspectos que los panelistas tienen en cuenta a la hora de aportar y priorizar las acciones de las enfermeras escolares, tal y como se refleja en los resultados.

Según los expertos, el tratamiento transversal que se le da a la EpS en las escuelas no es el adecuado. Coinciden con lo reflejado en diversos estudios e informes ${ }^{(7,9,21,}$ 23, ${ }^{30)}$ confirmando que las actuaciones aisladas y sin una intervención interdisciplinar adecuada, no disponen del grado de efectividad existente en proyectos coordinados y 
realizados a lo largo de la vida escolar. Comentan además, que ya han fracasado programas desarrollados en respuesta a una crisis puntual, con portavoces externos y poca implicación de la plantilla escolar, motivada posiblemente por falta de tiempo y recursos y escaso apoyo institucional educativo y sanitario; así como por la realización incoherente e inconexa de las actividades. Estas actividades aisladas, por tanto, no permiten adquirir hábitos además de no responder a nuevas necesidades.

Es necesario promulgar leyes que amparen la presencia de enfermeras escolares de manera generalizada y no únicamente de manera aislada en algunos centros ${ }^{(5,6,13)}$. Esta presencia física en los centros se ve refrendada por los panelistas, que mayoritariamente proponen y apoyan soluciones en base a su presencia regular en el mismo (Figura 1). En nuestro medio, esta situación de permanencia ya se produce, de forma aislada, en ciertos centros escolares, sobre todo, de titularidad privada/concertada.

Es indispensable tener en cuenta el ámbito escolar como un nuevo y diferenciado entorno de actuación, donde el papel y los conocimientos de las enfermeras pueden aportar mejoras en la salud de la comunidad educativa ${ }^{(5,16,23)}$. Nuestros participantes destacan que no se desarrolla por desconocimiento de los Servicios de Enfermería escolar y sus competencias, así como por la escasa implicación de las instituciones/legisladores, estando presente en el discurso de nuestros panelistas la carencia de recursos económicos, pero no siendo escogida como la principal causa. Docentes y padres participantes del estudio cualitativo de Nasser Laaoula ${ }^{(3)}$ creen que no se implanta esta figura por causas puramente económicas.

En la actualidad, existe un debate en torno a si la Enfermería Familiar y Comunitaria debe ser responsable de la Enfermería escolar o si se debe consolidar una figura propia $^{(3)}$. En vista de las áreas de conocimiento que creen los expertos que deberían tener la Enfermería escolar, se vierte la necesidad de creación de una nueva especialidad o perfil dentro de la profesión.

Hasta el momento no se han encontrado en la literatura otros estudios que hayan abordado dicha temática de modo similar. Esto hace que sea difícil establecer comparaciones con otros estudios e imposible analizar la evolución del fenómeno. Las principales fortalezas y limitaciones corresponden a las de cualquier estudio realizado bajo la técnica Delphi ${ }^{(18)}$. No existe la posibilidad de generalizar los datos obtenidos, aunque sí permite ofrecer opciones, tendencias y escenarios que sirvan de base para una futura actuación.

\section{CONCLUSIONES}

La enfermera escolar se convierte en un necesario agente de innovación en salud escolar que, en vista de los resultados de las acciones a desarrollar, no solo cuida al alumnado, sino a toda la comunidad educativa, en colaboración con otros profesionales de los centros escolares. Es por ello, que su presencia física debería, de algún modo, estar reglada en todos los centros educativos. Se intervendría en calidad de vida infantil y adolescente a través de programas de EpS y la detección precoz de problemas; además se trabajaría con familia y profesorado. De este modo, podría disminuir la incidencia de enfermedades prevalentes en nuestro medio y mejorar el estado de salud de toda la comunidad educativa, lo que conllevaría una escuela más inclusiva. 
Para que esto ocurra, necesitamos investigar sobre cómo definir, cuantificar y satisfacer las situaciones de salud y necesidades de la infancia y juventud en la escuela, así como del resto de la comunidad escolar, tanto a nivel nacional como regional. De este modo, se podrían ofrecer unos cuidados de excelencia, basados en la evidencia científica.

\section{REFERENCIAS}

1. Gallego de Pardo GP. Una estrategia de enfermería escolar en la promoción de la salud y prevención de la enfermedad. Aquichan [edición electrónica]. Oct 2003 [consultado 12 oct 2017]; 3 (3): 42-7. Disponible en: http://www.redalyc.org/articulo.oa?id=74130307

2. González García N, López Langa N. Antecedentes históricos y perfil de la enfermera escolar. Metas de Enfermería. Sep 2012; 15 (7): 50-4.

3. Nasser Laaoula AO. La Enfermería Escolar: Una necesidad sentida. Almería: Universidad de Almería. Repositorio Universidad de Almería. Dic 2011. Disponible en:

http://repositorio.ual.es/bitstream/handle/10835/466/La_enfermeria_escolar_una_n ecesidadsentida_Nasser_Laaoula_Ossama.pdf?sequence=1\&isAllowed=y.

4. López Gutiérrez I. Registro de intervenciones asistenciales de la enfermera escolar. Metas de Enfermería. Oct 2014; 17 (8): 55-9.

5. Guallart Samaniego P, Alemán Alcázar A, López Bonilla $C$, et al. Salud, Jóvenes y Enfermería Escolar. Una Revisión Bibliográfica. Paraninfo Digital. Nov 2014; 20.

6. Sánchez Moñino T. Educación para la salud en centros docentes no universitarios. La Figura del Profesional Sanitario en los Centros de Enseñanza: Enfermería Escolar. Enfermería Global [edición electrónica]. May 2005 [consultado 12 oct 2017]; 4 (6): [aprox. 2 p.]. Disponible en: http://revistas.um.es/eglobal/article/download/530/544.

7. Domínguez Aurrecoechea B. Educación para la salud en el ámbito escolar. En: AEPap (Asociación Española de Pediatría de Atención Primaria) ed. Taller de Educación para la salud en el ámbito escolar. Curso de Actualización Pediatría 2006. Madrid: Exlibris Ediciones. 2006. p. 187-200. Disponible en: https://www.aepap.org/sites/default/files/educacion_salud_escuela.pdf

8. Riquelme Pérez M. Educación para la salud escolar. En: AEPap (Asociación Española de Pediatría de Atención Primaria) ed. Taller de Educación para la salud en el ámbito escolar. Curso de Actualización Pediatría 2006. Madrid: Exlibris Ediciones. $2006 . \quad$ p. 185-6. Disponible en: https://www.aepap.org/sites/default/files/educacion_salud_escuela.pdf

9. Martínez Valero RM. Educación para la salud en los colegios por enfermeros. Investigación \& cuidados. Ene-Abr 2015; 13 (32): 32-4.

10. Carta de Ottawa para la Promoción de la Salud. $1^{\text {a }}$ Conferencia Internacional sobre promoción de la salud. Canadá (1986). Salud Publica Educ Salud [edición electrónica]. 2001[consultado 12 oct 2017]; 1 (1): 19-22. Disponible en: http://mpsp.webs.uvigo.es/rev01-1/Ottawa-01-1.pdf

11. Organización Mundial de la Salud. Declaración de Yakarta sobre Promoción de la Salud en el siglo XXI. Cuarta Conferencia Internacional sobre Promoción de la Salud: Nueva era, nuevos actores: Adaptar la Promoción de la Salud al siglo XXI. Yakarta República de Indonesia: Organización Mundial de la Salud. 21-25 jul 1997. Disponible en: http://www.who.int/healthpromotion/conferences/previous/jakarta/en/hpr_jakarta_d eclaration_sp.pdf. 
12. Asociación Madrileña de Enfermería en Centros Educativos (AMECE). Perfil de Enfermería Escolar. Madrid: Asociación Madrileña de Enfermería en Centros Educativos. 2010. Disponible en: http://www.amece.es/images/perfil.pdf

13. Millán Sáez AB. Actuación de Enfermería en un Colegio de Educación Especial, Enfermería: elemento clave. Investigación \& Cuidados [edición electrónica]. 2007 [consultado 12 oct 2017]; 5 (10): 6-14. Disponible en: http://www.bibliotecadigitalcecova.com/contenido/revistas/cat4/pdf/Revista_Investi gacion_y_Cuidados_N10.pdf

14. Llada Suárez R, Del Fresno Marqués L. Una aproximación rigurosa a la Enfermería Familiar y Comunitaria en el Servicio Nacional de Salud británico. La eficiencia como prioridad. RqR Enfermería Comunitaria (Revista SEAPA) [edición electrónica]. 2014 [consultado 12 oct 2017]; 2 (3): 24-35. Disponible en: https://dialnet.unirioja.es/servlet/articulo?codigo=5037328

15. Asociación Madrileña de Enfermería en Centros Educativos (AMECE). Competencias de Enfermería Escolar. Madrid: AMECE. 2015. Disponible en: http://www.codem.es/Adjuntos/CODEM/Documentos/Informaciones/Publico/2a572 c87-f198-4fbf-b327-1d8d4aece026/5C1B7237-DD0C-4891-8066-

60BD01570087/ec90aaac-9ca8-4712-9e81-df61619f3e43/ec90aaac-9ca8-47129e81-df61619f3e43.pdf

16. Ruiz Alcalá C, García Marina MC, González García N. Gestión de un servicio de Enfermería Escolar. Metas de Enfermería. 2012; 15 (8): 50-5.

17. Reguant Álvarez M, Torrado Fonseca M. El método Delphi. Revista d'Innovació i Recerca en Educació [edición electrónica]. 2016 [consultado 12 dic 2017]; 9 (1): 87-102. Disponible en: http://revistes.ub.edu/index.php/REIRE/article/download/reire2016.9.1916/18093

18. Varela-Ruiz M, Díaz-Bravo L, García-Durán R. Descripción y usos del método Delphi en investigaciones del área de la salud. Investigación en Educación Médica [edición electrónica]. 2012 [consultado 12 dic 2017]; 1 (2): 90-5. Disponible en: http://riem.facmed.unam.mx/sites/all/archivos/V1Num02/07_MI_DESCRIPCION_Y USOS.PDF

19. Díez Fernández T, Gómez Carrasco JA, Casas Martínez F, et al. ¿En qué medida es la enfermería escolar una necesidad sentida por los padres de los niños escolarizados en enseñanza primaria? Enfermería Clínica [edición electrónica]. 2001 [consultado 12 dic 2017]; 11 (2): 72-9. Disponible en: http://www.elsevier.es/es-revista-enfermeria-clinica-35-articulo-en-que-medida-esenfermeria-S1130862101736911.

20. Ministerio de Sanidad, Política Social e Igualdad. Los estilos de vida y la salud de los adolescentes españoles a lo largo de la primera década del milenio. Sevilla: Ministerio de Sanidad, Política Social e Igualdad. 2013. Disponible en: http://www.msssi.gob.es/profesionales/saludPublica/prevPromocion/promocion/sal udJovenes/estudioHBSC/docs/Comparativo2002a2010/Comparativo2002_2006_2 010.pdf

21. Ministerio de educación, política social y deporte, Ministerio de Sanidad y Consumo. Informe: Diagnóstico de situación sobre avances conseguidos, necesidades y retos en promoción y educación para la salud en la escuela en España. Madrid: Ministerio de Sanidad de Consumo. 2008. Disponible en: http://www.msssi.gob.es/profesionales/saludPublica/prevPromocion/promocion/sal udJovenes/docs/DiagnosticoSituacionEscuela.pdf

22. Davó-Blanes MC, García de la Hera M, La Parra D. Educación para la salud en la escuela primaria: opinión del profesorado de la ciudad de Alicante. Gac Sanit [edición electrónica]. 2016 [consultado 20 feb 2018]; 30 (1): 31-6. http://dx.doi.org/10.1016/j.gaceta.2015.07.008 
23. Mompeán Torrecillas PJ, Loba Abenza MV, Martínez García AV, et al. Educación para la salud en la etapa escolar como reto para docentes y sanitarios. Paraninfo Digital. 2017; 27: [aprox. 5 p.].

24. Santos Rego MA (Ed.). El poder de la familia en la educación. Madrid: Síntesis; 2015. $260 \mathrm{p}$.

25. Herrera Librero C, De Diego Cordero R. Importancia de la enfermería escolar en España. Paraninfo Digital. 2015; 22: [aprox. 5 p.].

26. Fernández-Morante C, Cebreiro López B, Fernández de la Iglesia JC, et al. Career counselling for childhood cancer survivors: a research study. Santiago de Compostela: Universidad de Santiago de Compostela. 2016.

27. Lima-Serrano M, Martínez-Montilla JM, Guerra-Martín MD, et al. Factores relacionados con la calidad de vida en la adolescencia. Gac Sanit [edición electrónica]. 2018 [consultado 1 mar 2018]; 32 (1): 68-71. http://dx.doi.org/10.1016/j.gaceta.2016.06.016

28. Costa Gueterres E, de Oliveira Rosa E, da Silveira A, et al. Educación para la salud en el contexto escolar: estudio de revisión integradora. Enfermería global [edición electrónica]. 2017 [consultado 20 feb 2018]; 16 (46): 464-99. Disponible en: http://revistas.um.es/eglobal/article/view/235801/210221

29. García Dueñas P, Cuesta del Amo M, González García N. Examen de salud básico en un servicio de Enfermería Escolar. Metas de Enfermería. 2012; 15 (9): 50-6.

30. Ministerio de Educación, política social y deporte, Ministerio de Sanidad y Consumo. Criterios de calidad para el desarrollo de proyectos y actuaciones de promoción y educación para la salud en el sistema educativo. Guía para las administraciones educativas y sanitarias. Madrid: Ministerio de Sanidad y Consumo. 2008.2 Disponible en: http://www.msssi.gob.es/profesionales/saludPublica/prevPromocion/promocion/sal udJovenes/docs/criteriosCalidad2MSC_MEC 